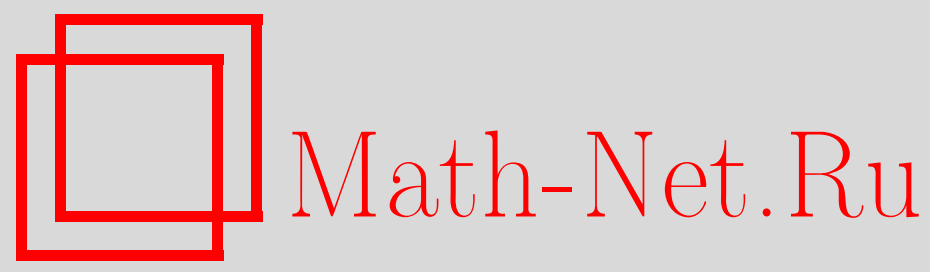

Е. И. Бережной, Подпространство пространства Гельдера, состоящее из самых негладких функций, Матем. заметки, 2003, том 74, выпуск 3, 329-339

DOI: https://doi.org/10.4213/mzm266

Использование Общероссийского математического портала Math-Net.Ru подразумевает, что вы прочитали и согласны с пользовательским соглашением http://www . mathnet.ru/rus/agreement

Параметры загрузки:

IP: 35.173 .219 .149

26 апреля 2023 г., $14: 17: 11$

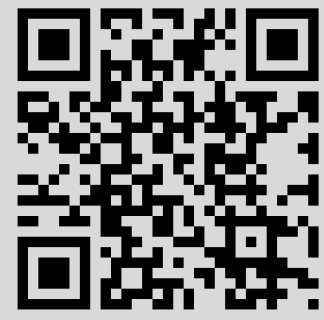




\title{
ПОДПРОСТРАНСТВО ПРОСТРАНСТВА ГЁЛЬДЕРА, СОСТОЯЩЕЕ ИЗ САМЫХ НЕГЛАДКИХ ФУНКЦИЙ
}

\author{
Е.И. Бережной
}

\begin{abstract}
В первой части работы дается полный ответ на старый вопрос из геометрической теории банаховых пространств, а именно, строится бесконечномерное замкнутое подпространство пространства Гёльдера такое, что у каждой отличной от тождественного нуля функции в каждой точке гладкость не лучше, чем у самой негладкой функции пространства Гёльдера. Во второй части работы, используя конструкции первой части, показано, что множество функций из пространства Гёльдера, гладкость которых на множестве положительной меры лучше, чем у самой негладкой функции, есть множество первой категории в этом пространстве.
\end{abstract}

Библиографоия: 10 названий.

В геометрической теории банаховых пространств гладких функций значительньй интерес представляет следующая задача (на нее снова обращалось внимание в [1]).

ЗАДАчА А. Пусть задано некоторое банахово пространство гладких функиий $Х$. Существует ли бесконечномерное замкнутое подпространство $Y \subset X$ такое, что у каждой отличной от тождественного нуля функиии $y \in Y$ гладкость не лучие, чем у самой негладкой функиии из $X$.

Этим вопросом занимались многие математики [1]-[5]. В первой части настоящей работы дается полное решение задачи А для пространств функций, удовлетворяющих условию Гёльдера. А именно, строится бесконечномерное замкнутое подпространство пространства Гёльдера такое, что у каждой отличной от тождественного нуля функции в каждой точке гладкость не лучше, чем у самой негладкой функции пространства Гёльдера.

Во второй части работы, используя конструкции первой части, показано, что множество функций из пространства Гёльдера, гладкость которых на множестве положительной меры лучше, чем у самой негладкой функции, есть множество первой категории. Из предшествующих работ по этой тематике отметим [6], в которой показано, что множество функций $x \in C[0,1]$ таких, что $\mu\{t: x(t)=y(t)\}=0(\mu(D)$ - мера Лебега множества $D$ ) для всех функций из фиксированного пространства Гёльдера, имеет вторую категорию в $C[0,1]$. В [7] приведен пример функции из $H_{\alpha}(0<\alpha<1)$ такой, что $\mu\{t: x(t)=y(t)\}=0$ для каждой функции из $H_{\beta}$ c $1 \geqslant \beta>\alpha$.

Работа выполнена при финансовой поддержке Российского фонда фундаментальных исследований, грант № 02-01-00428. 
Пусть $\omega:[0,1] \rightarrow \mathbb{R}_{+}$есть модуль непрерывности первого порядка, т.е. монотонно возрастающая, непрерьвная, полуаддитивная и равная нулю в нуле функция. Для модуля непрерьвности $\omega(t)$ через $\omega^{*}(t)$ обозначим наименьшую вогнутую мажоранту функции $\omega(t)$. Хорошо известно, что для всех $t \in[0,1]$ вьполняется следующее неравенство С. Б. Стечкина: $\omega(t) \leqslant \omega^{*}(t) \leqslant 2 \omega(t)$. Поэтому без ограничения обшности мы можем считать, что $\omega$ является вогнутой функцией. Через $H(\omega)$ обозначим банаховопространство функций на $[0,1]$, норма в котором определяется равенством

$$
\|x \mid H(\omega)\|=\max \left\{|x(0)|, \sup _{0 \leqslant t<s \leqslant 1} \frac{|x(t)-x(s)|}{\omega(|t-s|)}\right\},
$$

а через $H^{0}(\omega)$ обозначим подмножество $H(\omega)$, состоящее из функций $x$, для каждой из которых найдется своя точка $t_{x} \in(0,1)$, в которой справедливо хотя бы одно из соотношений

$$
\lim _{h \rightarrow+0} \frac{\left|x\left(t_{x}+h\right)-x\left(t_{x}\right)\right|}{\omega(h)}=0, \quad \lim _{h \rightarrow-0} \frac{\left|x\left(t_{x}+h\right)-x\left(t_{x}\right)\right|}{\omega(|h|)}=0 .
$$

Если $\omega(h)=h^{\alpha}(0<\alpha \leqslant 1)$, то соответствующее пространство обозначается через $H_{\alpha}$.

Основным результатом первой части настоящей статьи является

ТЕОРемА 1. Пусть модуль непрерывности $\omega$ удовлетворяет соотношению

$$
\lim _{h \rightarrow 0} \frac{\omega(h)}{h}=\infty
$$

Тогда существует замкнутое бесконечномерное подпространство $B \subset H(\omega)$ такое, что каждая отличная от тождественного нуля функиия $x \in B$ не принадлехит $H^{0}(\omega)$.

ЗАмЕчАНИЕ 1. Отметим, что условие (1) и любое из условий

$$
\varliminf_{h \rightarrow 0} \frac{\omega(h)}{h}=\infty, \quad \varlimsup_{h \rightarrow 0} \frac{\omega(h)}{h}=\infty
$$

эквивалентны. В самом деле, из вогнутости функции $\omega(t)$ следует, что отношение $\omega(t) / t$ не возрастает. Поэтому (1) и любое из $\left(1^{\prime}\right)$ эквивалентны.

Для доказательства теоремы нам потребуются некоторые вспомогательные построения.

Для модуля непрерьвности $\omega(h)$, удовлетворяющего (1), можно определить так назьваемую заполняющую последовательность. Эта последовательность строится так.

Положим $h_{0}=1$ и пусть точки $h_{1}, \ldots, h_{n}$ определены. Определим теперь точку $h_{n+1}$ равенством

$$
h_{n+1}=\sup \left\{t: \frac{\omega(t)}{t} \geqslant 100 \frac{\omega\left(h_{n}\right)}{h_{n}} \& \omega(t) \leqslant \frac{1}{100} \omega\left(h_{n}\right)\right\} .
$$

Прямо из (2) следует, что для каждого $k \in \mathbb{N}$ вьполняются неравенства

$$
\begin{aligned}
& \sum_{j=0}^{k-1} \frac{\omega\left(h_{j}\right)}{h_{j}} \leqslant \frac{100}{99} \frac{\omega\left(h_{k-1}\right)}{h_{k-1}} \leqslant \frac{1}{99} \frac{\omega\left(h_{k}\right)}{h_{k}}, \quad h_{k+1} \leqslant \frac{h_{k}}{100}, \\
& \sum_{j=k+1}^{\infty} \omega\left(h_{j}\right) \leqslant \frac{100}{99} \omega\left(h_{k+1}\right) \leqslant \frac{1}{99} \omega\left(h_{k}\right) .
\end{aligned}
$$


Заполняющие последовательности в последнее время находят все большее применение в различных областях анализа (см., например, [8]-[10]).

Непосредственно из (3) следует основное свойство заполняющих последовательностей.

ЛЕмма 1. Положим

$$
\bar{\omega}(t)=\sum_{j=0}^{\infty} \omega\left(h_{j}\right) \min \left\{\frac{t}{h_{j}}, 1\right\} .
$$

Тогда для $t \in\left(h_{n+1}, h_{n}\right]$ справедливы соотношения

$$
\begin{aligned}
t \frac{\omega\left(h_{n}\right)}{h_{n}}+\omega\left(h_{n+1}\right) & \leqslant \bar{\omega}(t) \leqslant \frac{100}{99}\left(t \frac{\omega\left(h_{n}\right)}{h_{n}}+\omega\left(h_{n+1}\right)\right), \\
\frac{1}{100} \omega(t) & \leqslant \bar{\omega}(t) \leqslant \frac{200}{99} \omega(t) .
\end{aligned}
$$

Положим

$$
\varphi_{j}(t)= \begin{cases}\omega\left(h_{j}\right) \frac{t}{h_{j}}, & \text { при } t \in\left[0, h_{j}\right], \\ \omega\left(h_{j}\right)\left(2-\frac{t}{h_{j}}\right), & \text { при } t \in\left(h_{j}, 2 h_{j}\right],\end{cases}
$$

и продолжим $\varphi_{j}$ на всю ось периодически с помощью равенства $\varphi_{j}\left(t+2 h_{j}\right)=\varphi_{j}(t)$.

Легко проверить, что при всех $t \in \mathbb{R}, h>0$ справедливо неравенство

$$
\left|\varphi_{j}(t+h)-\varphi_{j}(t)\right| \leqslant \omega\left(h_{j}\right) \min \left\{\frac{h}{h_{j}}, 1\right\} .
$$

Если же при некотором целом $k$ выполнены неравенства $k h_{j} \leqslant t<t+h \leqslant(k+1) h_{j}$, то справедливо равенство

$$
\left|\varphi_{j}(t+h)-\varphi_{j}(t)\right|=\omega\left(h_{j}\right) \frac{h}{h_{j}} .
$$

Занумеруем простые числа натурального ряда, начиная с 2 , в последовательность $\left\{p_{i}\right\}_{1}^{\infty}$. Для каждого $k \in \mathbb{N}$ положим

$$
\psi_{k}(t)=\sum_{j=1}^{\infty} \varphi_{\left(p_{k}\right)^{j}}(t) .
$$

Из (3) и (6) следует, что ряд в правой части (9) абсолютно сходится в пространстве непрерьвных функций $C[0,1]$. Кроме того, из $(7),(8)$ и леммы 1 получим неравенство

$$
\begin{aligned}
\left|\psi_{k}(t+h)-\psi_{k}(t)\right| & \leqslant \sum_{j=1}^{\infty}\left|\varphi_{\left(p_{k}\right)^{j}}(t+h)-\varphi_{\left(p_{k}\right)^{j}}(t)\right| \\
& \leqslant \sum_{j=1}^{\infty} \omega\left(h_{\left(p_{k}\right)^{j}}\right) \min \left\{\frac{h}{h_{\left(p_{k}\right)^{j}}}, 1\right\} \leqslant \bar{\omega}(h) \leqslant \frac{200}{99} \omega(h) .
\end{aligned}
$$


С другой стороны, из (3) и $(8)$ для $\bar{h}=h_{\left(p_{k}\right)^{j}}$ получим

$$
\begin{aligned}
\left|\psi_{k}(\bar{h})-\psi_{k}(0)\right|=\left|\psi_{k}(\bar{h})\right| & \geqslant\left|\varphi_{\left(p_{k}\right)^{j}}(\bar{h})\right|-\sum_{i \neq j}\left|\varphi_{\left(p_{k}\right)^{i}}(\bar{h})\right| \\
& \geqslant \omega(\bar{h})-\left(\sum_{i<\left(p_{k}\right)^{j}}\left|\varphi_{i}(\bar{h})\right|+\sum_{i>\left(p_{k}\right)^{j}}\left|\varphi_{i}(\bar{h})\right|\right) \\
& \geqslant \omega(\bar{h})-\left(\frac{1}{99} \omega(\bar{h})+\frac{1}{99} \omega(\bar{h})\right)=\frac{97}{99} \omega(\bar{h}) .
\end{aligned}
$$

Таким образом, из (10) и (11) следуют важные соотношения:

$$
\frac{97}{99} \leqslant\left\|\psi_{k} \mid H(\omega)\right\|<\frac{200}{99} .
$$

ЛЕмма 2. Пусть задана последовательность $\left\{b_{i}\right\}_{1}^{\infty} \in l^{\infty}$. Положсим

$$
x(t)=\sum_{i=1}^{\infty} b_{i} \psi_{i}(t) .
$$

Тогда $x \in H(\omega)$ и справедливы неравенства

$$
\frac{95}{99} \sup _{i}\left|b_{i}\right| \leqslant\left\|x\left|H(\omega) \| \leqslant \frac{200}{99} \sup _{i}\right| b_{i} \mid .\right.
$$

ДокАЗАТЕЛЬСТво. Из (3) следует, что ряд, определяющий функцию $x(t)$, равномерно сходится в $C[0,1]$. Используя $(3),(5)$ и $(7)$ докажем правое неравенство в $(13)$. Имеем

$$
\begin{aligned}
|x(t+h)-x(t)| & =\left|\sum_{i=1}^{\infty} b_{i}\left(\psi_{i}(t+h)-\psi_{i}(t)\right)\right| \leqslant \sup _{i}\left|b_{i}\right| \sum_{k=1}^{\infty}\left|\psi_{k}(t+h)-\psi_{k}(t)\right| \\
& \leqslant \sup _{i}\left|b_{i}\right| \sum_{k=1}^{\infty} \sum_{j=1}^{\infty}\left|\varphi_{\left(p_{k}\right)^{j}}(t+h)-\varphi_{\left(p_{k}\right)^{j}}(t)\right| \\
& \leqslant \sup _{i}\left|b_{i}\right| \sum_{j=1}^{\infty}\left|\varphi_{j}(t+h)-\varphi_{j}(t)\right| \leqslant \sup _{i}\left|b_{i}\right| \sum_{j=1}^{\infty} \omega\left(h_{j}\right) \min \left\{\frac{h}{h_{j}}, 1\right\} \\
& =\bar{\omega}(h) \sup _{i}\left|b_{i}\right| \leqslant \frac{200}{99} \omega(h) \sup _{i}\left|b_{i}\right| .
\end{aligned}
$$

Докажем левое неравенство в (13). Положим $\bar{h}=h_{\left(p_{k}\right)^{j}}, m=\left(p_{k}\right)^{j}$. Тогда из (11) получим

$$
\begin{aligned}
|x(\bar{h})-x(0)| & =\left|\sum_{i=1}^{\infty} b_{i} \psi_{i}(\bar{h})\right| \geqslant\left|b_{k}\right|\left|\psi_{k}(\bar{h})\right|-\sum_{i \neq k} b_{i}\left|\psi_{i}(\bar{h})\right| \\
& \geqslant \frac{97}{99}\left|b_{k}\right| \omega(\bar{h})-\sup _{i \neq k} b_{i} \sum_{i \neq k}\left|\psi_{i}(\bar{h})\right| \\
& \geqslant \frac{97}{99}\left|b_{k}\right| \omega(\bar{h})-\sup _{i \neq k} b_{i}\left(\sum_{i<m}\left|\psi_{i}(\bar{h})\right|+\sum_{i>m}\left|\psi_{i}(\bar{h})\right|\right) \\
& \geqslant \frac{97}{99}\left|b_{k}\right| \omega(\bar{h})-\sup _{i \neq k} b_{i}\left(\frac{1}{99} \omega(\bar{h})+\frac{1}{99} \omega(\bar{h})\right)=\frac{1}{99} \omega(\bar{h})\left(97\left|b_{k}\right|-2 \sup _{i \neq k} b_{i}\right) .
\end{aligned}
$$

Лемма доказана. 
ДокАЗАТЕльСтво тЕоремы 1 . Положим $\left.B=\overline{\operatorname{span}\left\{\psi_{i} /\left\|\psi_{i} \mid H(\omega)\right\|\right.}\right\}_{i=1}^{\infty}$. Тогда из (12) и леммы 2 следует, что пространство $B$ является замкнутым подпространством пространства $H(\omega)$ изоморфным пространству $l^{\infty}$, причем базис $\left\{\psi_{i} /\left\|\psi_{i} \mid H(\omega)\right\|\right\}_{i=1}^{\infty}$ эквивалентен единичному базису пространства $l^{\infty}$.

Пусть задана функция $x(t)=\sum_{i=j}^{\infty} a_{i} \psi_{i}(t)$, для которой $a_{j} \neq 0$ и

$$
\sup _{i}\left|a_{i}\right|=1 .
$$

Покажем, что в каждой точке $t_{0} \in(0,1)$ выполнено соотношение

$$
\varlimsup_{h \rightarrow+0} \frac{\left|x\left(t_{0}+h\right)-x\left(t_{0}\right)\right|}{\omega(h)}>0 .
$$

Зафиксируем $i_{0} \in \mathbb{N}$ так, чтобы $\left|a_{i_{0}}\right| \geqslant 7 / 8$, и рассмотрим функцию $\psi_{i_{0}}$. Выберем $k \in \mathbb{N}$, обозначим $j_{0}=\left(p_{i_{0}}\right)^{k}$ и выберем точку $t_{1}$ так, чтобы $h_{j_{0}} / 8 \geqslant t_{0}-t_{1}>h_{j_{0}} / 16$. Если при некотором $l$ вьполнены неравенства

$$
l h_{j_{0}} \leqslant t_{0}<t_{1} \leqslant(l+1) h_{j_{0}}
$$

то согласно (6), (8) и (14) справедлива оценка

$$
\begin{aligned}
\left|x\left(t_{0}\right)-x\left(t_{1}\right)\right| & \geqslant\left|a_{i_{0}}\right|\left|\psi_{i_{0}}\left(t_{0}\right)-\psi_{i_{0}}\left(t_{1}\right)\right|-\sum_{i \neq i_{0}}\left|a_{i}\right|\left|\psi_{i}\left(t_{0}\right)-\psi_{i}\left(t_{1}\right)\right| \\
& \geqslant \frac{7}{8}\left|\varphi_{j_{0}}\left(t_{0}\right)-\varphi_{j_{0}}\left(t_{1}\right)\right|-\sum_{j \neq j_{0}}\left|\varphi_{j}\left(t_{0}\right)-\varphi_{j}\left(t_{1}\right)\right| \\
& \geqslant \frac{7}{8}\left|\varphi_{j_{0}}\left(t_{0}\right)-\varphi_{j_{0}}\left(t_{1}\right)\right|-\left|t_{0}-t_{1}\right| \sum_{j<j_{0}} \frac{\omega\left(h_{j}\right)}{h_{j}}-\sum_{j>j_{0}} \omega\left(h_{j}\right) \\
& \geqslant\left(\frac{7}{8}-\frac{1}{99}\right)\left|t_{0}-t_{1}\right| \frac{\omega\left(h_{j_{0}}\right)}{h_{j_{0}}}-\frac{1}{99} \omega\left(h_{j_{0}}\right) \\
& \geqslant\left(\left(\frac{7}{8}-\frac{1}{99}\right) \frac{1}{16}-\frac{1}{99}\right) \omega\left(h_{j_{0}}\right) \geqslant \frac{1}{32} \omega\left(h_{j_{0}}\right),
\end{aligned}
$$

и поэтому справедливо неравенство

$$
\frac{\left|x\left(t_{0}\right)-x\left(t_{1}\right)\right|}{\omega\left(\left|t_{0}-t_{1}\right|\right)} \geqslant \frac{1}{32} .
$$

Если соотношения (16) нарушаются, то положим $t_{1}=t_{0}+h_{j_{0}}$. Из геометрических соображений легко видеть, что в этом случае для ключевого слагаемого $\left|\varphi_{j_{0}}\left(t_{0}\right)-\varphi_{j_{0}}\left(t_{1}\right)\right|$ справедлива оценка

$$
\begin{aligned}
\left|\varphi_{j_{0}}\left(t_{0}\right)-\varphi_{j_{0}}\left(t_{1}\right)\right| & \geqslant\left|\varphi_{j_{0}}\left(t_{1}\right)-\varphi_{j_{0}}\left((l+1) h_{j_{0}}\right)\right|-\left|\varphi_{j_{0}}\left(t_{0}\right)-\varphi_{j_{0}}\left((l+1) h_{j_{0}}\right)\right| \\
& \geqslant \frac{\omega\left(h_{j_{0}}\right)}{h_{j_{0}}}\left(t_{1}-(l+1) h_{j_{0}}\right)-\frac{\omega\left(h_{j_{0}}\right)}{h_{j_{0}}}\left((l+1) h_{j_{0}}-t_{0}\right) \\
& \geqslant \frac{15}{16} \omega\left(h_{j_{0}}\right)-\frac{1}{16} \omega\left(h_{j_{0}}\right)=\frac{7}{8} \omega\left(h_{j_{0}}\right)=\frac{7}{8} \omega\left(\left|t_{1}-t_{0}\right|\right),
\end{aligned}
$$


и поэтому справедливо неравенство

$$
\frac{\left|x\left(t_{0}\right)-x\left(t_{1}\right)\right|}{\omega\left(\left|t_{0}-t_{1}\right|\right)} \geqslant\left(\frac{7}{8}\right)^{2}-\frac{2}{99}>\frac{5}{8} .
$$

Из (17) и (18) следует, что вьполнено (15).

Выполнение соотношения

$$
\lim _{h \rightarrow-0} \frac{\left|x\left(t_{0}+h\right)-x\left(t_{0}\right)\right|}{\omega(|h|)} \neq 0
$$

объясняется аналогично.

Теорема доказана.

ЗАмЕчАниЕ 2. Предположим теперь, что для модуля непрерьвности $\omega_{0}(h)$ условие (1) не вьполнено. Это означает, что $\omega_{0}(h)$ эквивалентно $h$. Покажем, что в этом случае нет аналога теоремы 1. Действительно, любая функция из $H_{1}$ абсолютно непрерьвна, и поэтому дифференцируема почти всюду. Пусть теперь $x, y \in H_{1}$. Если найдется $t_{1}$, для которой $y^{\prime}\left(t_{1}\right)=0$, то $y \in H_{1}^{0}$; в противном случае выберем точку $t_{0} \in(0,1)$, в которой существуют производные у обеих функций, и рассмотрим линейную комбинацию $z(t)=$ $x(t)-\left(x^{\prime}\left(t_{0}\right) / y^{\prime}\left(t_{0}\right)\right) y(t)$. Тогда $z \in H_{1}^{0}$. Таким образом, в случае нарушения условия (1) аналога теоремы 1 не может быть.

Сейчас мы продемонстрируем, как построенные вьше функции могут быть применены в задаче об исправимости функций из $H(\omega)$.

ОПРЕДЕЛЕНИЕ 1. Будем говорить, что функция $x \in H(\omega)$ исправима, если существует измеримое множество $M$ положительной меры такое, что для всех $t \in M$ вьполнено соотношение

$$
\lim _{s \rightarrow t ; s, t \in M} \frac{|x(t)-x(s)|}{\omega(|t-s|)}=0 .
$$

Отметим, что если продолжить функцию $x$ с множества $M$, о котором говорится в определении 1 , на замыкание $M$ по непрерывности, а потом продолжить на $[0,1] \backslash M$ по линейности, то у продолженной функции гладкость будет вьше, чем у самой негладкой функции из $H(\omega)$.

Следующая теорема демонстрирует “равномерное” отсутствие свойства исправимости у функций из пространства $B$, построенного выше.

Теорема 2. Пусть В подпространство $H(\omega)$, построенное в теореме 1 . Тогда каждая отличная от тождественного нуля функиия $x \in B$ не является исправимой. Более того, существует константа $c_{0}>0$ такая, что для каждой функиии $x \in B$ c $\|x \mid H(\omega)\|=1$ и произвольного множества $M$ положительной мерь найдется последовательность пар точек $t_{0, n}, t_{1, n} \in M,\left|t_{0, n}-t_{1, n}\right| \rightarrow 0$ при $n \rightarrow \infty$, для которой

$$
\left|x\left(t_{0, n}\right)-x\left(t_{1, n}\right)\right| \geqslant c_{0} \omega\left(\left|t_{0, n}-t_{1, n}\right|\right) .
$$

Можно полохить $c_{0}=1 / 32$.

ДокАЗАТЕЛЬСТво теоремы 2 аналогично доказательству теоремы 1 . Только вместо точек $t_{0}, t_{1}$ в доказательстве основной теоремы нужно использовать пару точек, существование которых гарантируется следующей элементарной леммой о точках плотности. 
Лемма 3. Пусть $t_{0}$ есть точка плотности измеримого множества D. Тогда существует такое полохительное число $\delta\left(t_{0}\right)$, что при каждом $\delta: 0<\delta<\delta\left(t_{0}\right)$ существуют две точки $t_{1}^{\prime} \in D, t_{1}^{\prime \prime} \in D$ такие, что выполняются неравенства

$$
\frac{\delta}{8} \geqslant t_{0}-t_{1}^{\prime} \geqslant \frac{\delta}{16}, \quad \frac{\delta}{8} \geqslant t_{1}^{\prime \prime}-t_{0} \geqslant \frac{\delta}{16} \text {. }
$$

Следующие определения связаны с аппроксимативной гладкостью функции $x \in H(\omega)$ относительно модуля непрерьвности $\omega$.

ОПРЕДЕЛЕНИЕ 2. Пусть функция $x \in H(\omega)$. Будем говорить, что точка $t \in(0,1)$ принадлежит множеству $M_{x}$, если на некотором множестве $M$, для которого точка $t$ является точкой плотности, вьполняется соотношение

$$
\lim _{s \rightarrow t ; s \in M} \frac{|x(t)-x(s)|}{\omega(|t-s|)}=0 .
$$

Если $\mu\left(M_{x}\right)>0$, то будем говорить, что $x$ принадлежсит классу $H_{a p}^{0}(\omega)$.

ОПРЕДЕЛЕНИЕ 3. БУдем говорить, что точка $t \in(0,1)$ принадлежит множеству $M_{x, n}$, если для любого подмножества $M$, имеющего точку $t$ точкой плотности, вьполняется соотношение

$$
\varlimsup_{s \rightarrow t ; s \in M} \frac{|x(t)-x(s)|}{\omega(|t-s|)} \geqslant \frac{1}{n} .
$$

Для функции $x \in H(\omega)$ свойства множеств $M_{x}$ и $M_{x, n}$ собраны в следующей лемме. Лемма 4. Пусть $x \in H(\omega)$. Тогда справедливы следующие соотношения:

$$
\begin{gathered}
M_{x, n+1} \subseteq M_{x, n}, \\
M_{x} \cap\left(\bigcup_{n=1}^{\infty} M_{x, n}\right)=\varnothing, \\
\mu\left(M_{x}\right)+\mu\left(\bigcup_{n=1}^{\infty} M_{x, n}\right)=1 .
\end{gathered}
$$

ДокАЗАТЕЛЬСтво. Вьполнение свойств (19) и (20) очевидно. Докажем (21).

Пусть $t$ является точкой плотности множества $(0,1) \backslash\left(\bigcup_{n=1}^{\infty} M_{x, n}\right)$. Покажем, что $t \in M_{x}$. Так как $t$ является точкой плотности множества $(0,1) \backslash\left(\bigcup_{n=1}^{\infty} M_{x, n}\right)$, найдется последовательность множеств $M_{n}$, для каждого из которых точка $t$ является точкой плотности и для каждого из которых выполнено неравенство

$$
\sup _{s \in M_{n}} \frac{|x(t)-x(s)|}{\omega(|t-s|)} \leqslant \frac{1}{n} .
$$

Выберем сначала последовательность $\left\{\epsilon_{i}\right\}$ так, чтобы выполнялись условия

$$
0<\epsilon_{i+1}<\epsilon_{i}, \quad \lim _{k \rightarrow \infty} \prod_{i=k}^{\infty}\left(1-\epsilon_{i}\right)=1 .
$$


Далее построим последовательность $\left\{\delta_{i}\right\}$. Сначала выберем $\delta_{1}>0$ так, чтобы при всех $0<\delta<\delta_{1}$ вьполнялось неравенство

$$
\mu\left(M_{1} \cap(t-\delta, t+\delta)\right)>2\left(1-2^{-1}\right) \delta
$$

Положим $I_{1}=\left(t-\delta_{1}, t+\delta_{1}\right)$, и пусть элементы $\delta_{2}, \delta_{3}, \ldots, \delta_{n}$ и интервалы $I_{2}=(t-$ $\left.\delta_{2}, t+\delta_{2}\right), \ldots, I_{n}=\left(t-\delta_{n}, t+\delta_{n}\right)$ уже построены. Выберем теперь $\delta_{n+1} \in\left(0, \delta_{n}\right)$ так, чтобы при всех $0<\delta<\delta_{n+1}$ выполнялись следующие условия:

$$
\begin{aligned}
\mu\left(M_{n+1} \cap(t-\delta, t+\delta)\right) & >2\left(1-2^{-n-1}\right) \delta, \\
\mu\left(M_{n+1} \cap I_{n+1}\right) & >\left(1-\epsilon_{n}\right) \mu\left(M_{n} \cap I_{n+1}\right) .
\end{aligned}
$$

Для всех $n \in \mathbb{N}$ положим $C_{n}=\left(t-\delta_{n}, t-\delta_{n+1}\right) \cup\left(t+\delta_{n+1}, t+\delta_{n}\right)$ и определим множество $M$ равенством

$$
M=t \cup\left(\bigcup_{n=1}^{\infty}\left(M_{n} \cap C_{n}\right)\right) .
$$

Покажем сначала, что $t$ есть точка плотности множества $M$.

Пусть $\delta \in\left[\delta_{n+1}, \delta_{n}\right)$. Оценим меру множества $M \cap(t-\delta, t+\delta)$. Из представления $(t-\delta, t+\delta)=\left(t-\delta, t-\delta_{n+1}\right) \cup\left(t+\delta_{n+1}, t+\delta\right) \cup\left(\bigcup_{k=n+1}^{\infty} C_{k}\right)$ получим

$$
\begin{aligned}
\mu(M \cap(t-\delta, t+\delta))= & \mu\left(M_{n} \cap\left\{\left(t-\delta, t-\delta_{n+1}\right) \cup\left(t+\delta_{n+1}, t+\delta\right)\right\}\right) \\
& +\sum_{k=n+1}^{\infty} \mu\left(M_{k} \cap C_{k}\right) .
\end{aligned}
$$

Используя (24), получим неравенство

$$
\begin{aligned}
& \sum_{k=n+1}^{m} \mu\left(M_{k} \cap C_{k}\right)+\mu\left(M_{m} \cap I_{m+1}\right)=\sum_{k=n+1}^{m-1} \mu\left(M_{k} \cap C_{k}\right)+\mu\left(M_{m} \cap I_{m}\right) \\
& \quad=\sum_{k=n+1}^{m-2} \mu\left(M_{k} \cap C_{k}\right)+\mu\left(M_{m-1} \cap C_{m-1}\right)+\mu\left(M_{m} \cap I_{m}\right) \\
& \geqslant \sum_{k=n+1}^{m-2} \mu\left(M_{k} \cap C_{k}\right)+\mu\left(M_{m-1} \cap C_{m-1}\right)+\left(1-\epsilon_{m-1}\right) \mu\left(M_{m-1} \cap I_{m}\right) \\
& \geqslant \sum_{k=n+1}^{m-2} \mu\left(M_{k} \cap C_{k}\right)+\left(1-\epsilon_{m-1}\right) \mu\left(M_{m-1} \cap I_{m-1}\right) \\
& \geqslant \sum_{k=n+1}^{m-3} \mu\left(M_{k} \cap C_{k}\right)+\left(1-\epsilon_{m-2}\right)\left(1-\epsilon_{m-1}\right) \mu\left(M_{m-2} \cap I_{m-2}\right) \\
& \geqslant \mu\left(M_{n+1} \cap C_{n+1}\right)+\mu\left(M_{n+2} \cap I_{n+2}\right) \prod_{k=n+2}^{m-1}\left(1-\epsilon_{k}\right) \\
& \geqslant \mu\left(M_{n+1} \cap I_{n+1}\right) \prod_{k=n+1}^{m-1}\left(1-\epsilon_{k}\right) . \quad(26)
\end{aligned}
$$


Поэтому из (24)-(26) следует, что верно и такое неравенство:

$$
\begin{aligned}
\mu(M \cap(t-\delta, t+\delta)) \geqslant & \mu\left(M_{n} \cap\left\{\left(t-\delta, t-\delta_{n+1}\right) \cup\left(t+\delta_{n+1}, t+\delta\right)\right\}\right) \\
& +\mu\left(M_{n+1} \cap I_{n+1}\right) \prod_{k=n+1}^{\infty}\left(1-\epsilon_{k}\right) \\
\geqslant & \mu\left(M_{n} \cap\left\{\left(t-\delta, t-\delta_{n+1}\right) \cup\left(t+\delta_{n+1}, t+\delta\right)\right\}\right) \\
& +\mu\left(M_{n} \cap I_{n+1}\right) \prod_{k=n}^{\infty}\left(1-\epsilon_{k}\right) \\
\geqslant & \mu\left(M_{n} \cap(t-\delta, t+\delta)\right) \prod_{k=n}^{\infty}\left(1-\epsilon_{k}\right) \\
\geqslant & 2 \delta\left(1-2^{-n}\right) \prod_{k=n}^{\infty}\left(1-\epsilon_{k}\right) .
\end{aligned}
$$

Из последнего неравенства и (23) непосредственно следует, что $t$ является точкой плотности множества $M$.

Равенство

$$
\lim _{s \rightarrow t ; s \in M} \frac{|x(s)-x(t)|}{\omega(|s-t|)}=0
$$

следует из (22):

$$
\lim _{s \rightarrow t ; s \in M} \frac{|x(s)-x(t)|}{\omega(|s-t|)} \leqslant \lim _{n \rightarrow \infty} \sup _{s \in\left(M_{n} \cap C_{n}\right)} \frac{|x(s)-x(t)|}{\omega(|s-t|)} \leqslant \lim _{n \rightarrow \infty} \frac{1}{n}=0 .
$$

Таким образом $t \in M_{x}$. Лемма доказана.

Покажем теперь, что множество функций $H_{a p}^{0}(\omega)$ является множеством первой категории в $H(\omega)$.

ТЕОрема 3. Пусть $\omega$-некоторый модуль непрерывности. Тогда множество функиий $H_{a p}^{0}(\omega)$ является мнохеством первой категории в $H(\omega)$.

ДокАЗАТЕЛЬСтво. Предположим сначала, что модуль непрерьвности удовлетворяет условию (1).

Определим для $n=2,3, \ldots$ множества $U_{n}(\omega) \subset H(\omega)$ с помощю равенств

$$
U_{n}(\omega)=\left\{x \in H(\omega): \frac{1}{n}<\mu\left(M_{x}\right) \leqslant \frac{1}{n-1}\right\} .
$$

Покажем, что каждое множество $U_{n}(\omega)$ является нигде не плотным в $H(\omega)$. Для этого достаточно показать, что любой шар с центром в точке $x_{0} \in U_{n}(\omega)$ содержит шар из $H(\omega)$ без точек множества $U_{n}(\omega)$.

Итак, пусть задан шар $B\left(x_{0}, R\right) \subset U_{n}(\omega)$. Для каждого $m=2,3, \ldots$ образуем множества $M_{x_{0}, m}$. Согласно лемме 4 выберем теперь $m_{0}$ так, чтобы вьполнялось неравенство

$$
m\left(M_{x_{0}} \cup M_{x_{0}, m_{0}}\right) \geqslant 1-\frac{1}{n^{2}} .
$$


Положим $\bar{\psi}=\psi_{1} /\left\|\psi_{1} \mid H(\omega)\right\|$ (функции $\psi_{k}$ определены равенством $\left.(9)\right)$. Тогда $\bar{\psi} \epsilon$ $B$ и поэтому для нее справедлива вторая половина теоремы 2 с некоторой константой $c_{0}>0$. Выберем теперь $\epsilon>0$ так, чтобы выполнялись неравенства

$$
\epsilon\left(1+\frac{c_{0}}{2}\right)<R, \quad 0<\frac{1}{m_{0}}-\epsilon\left(1+c_{0}\right)
$$

Образуем функцию $x_{1}=x_{0}+\epsilon \bar{\psi}$ и рассмотрим шар $B\left(x_{1}, \epsilon c_{0} / 2\right)$. Из первого условия в (28) следует, что $B\left(x_{1}, \epsilon c_{0} / 2\right) \subseteq B\left(x_{0}, R\right)$. Покажем, что каждая функция $z \in$ $B\left(x_{1}, \epsilon c_{0} / 2\right)$ не принадлежит множеству $U_{n}(\omega)$.

Предположим противное. Тогда из (27) следует, что $\mu\left(\left\{M_{x_{0}} \cup M_{x_{0}, m_{0}}\right\} \cap M_{z}\right)>0$. Так как $M_{x_{0}} \cap M_{x_{0}, m_{0}}=\varnothing$, возможны два случая.

Рассмотрим первый случай. Пусть $\mu\left(M_{x_{0}} \cap M_{z}\right)>0$. Применим теорему 2 для множества $M_{x_{0}} \cap M_{z}$. Согласно теореме 2 найдется последовательность пар точек $t_{0, k}, t_{1, k}$ из $M_{x_{0}} \cap M_{z}$ такая, что $\left|t_{0, k}-t_{1, k}\right| \rightarrow 0$ при $k \rightarrow \infty$, и выполнены соотношения

$$
\begin{aligned}
\varlimsup_{k \rightarrow \infty} \frac{\left|z\left(t_{0, k}\right)-z\left(t_{1, k}\right)\right|}{\omega\left(\left|t_{0, k}-t_{1, k}\right|\right)} & \geqslant \varlimsup_{k \rightarrow \infty} \frac{\left|x_{1}\left(t_{0, k}\right)-x_{1}\left(t_{1, k}\right)\right|-\epsilon \frac{c_{0}}{2} \omega\left(\left|t_{0, k}-t_{1, k}\right|\right)}{\omega\left(\left|t_{0, k}-t_{1, k}\right|\right)} \\
& \geqslant \epsilon \varlimsup_{k \rightarrow \infty} \frac{\left|\bar{\psi}\left(t_{0, k}\right)-\bar{\psi}\left(t_{1, k}\right)\right|}{\omega\left(\left|t_{0, k}-t_{1, k}\right|\right)}-\varlimsup_{k \rightarrow \infty} \frac{\left|x_{0}\left(t_{0, k}\right)-x_{0}\left(t_{1, k}\right)\right|}{\omega\left(\left|t_{0, k}-t_{1, k}\right|\right)}-\epsilon \frac{c_{0}}{2} \\
& \geqslant \epsilon c_{0}-\epsilon \frac{c_{0}}{2}=\epsilon \frac{c_{0}}{2}>0,
\end{aligned}
$$

что противоречит тому, что $t_{0, k}, t_{1, k} \in M_{z}$.

Пусть теперь $\mu\left(M_{x_{0}, m_{0}} \cap M_{z}\right)>0$. Тогда для любой точки плотности $t \in M_{x_{0}, m_{0}} \cap$ $M_{z}$ прямо из определения следует неравенство

$$
\begin{aligned}
|z(t)-z(s)| & \geqslant\left|x_{1}(t)-x_{1}(s)\right|-\frac{\epsilon c_{0}}{2} \omega(|t-s|) \\
& \geqslant\left|x_{0}(t)-x_{0}(s)\right|-\epsilon|\bar{\psi}(t)-\bar{\psi}(s)|-\frac{\epsilon c_{0}}{2} \omega(|t-s|) \\
& \geqslant \frac{1}{m_{0}} \omega(|t-s|)-\epsilon \omega(|t-s|)-\frac{\epsilon c_{0}}{2} \omega(|t-s|) \\
& \geqslant\left(\frac{1}{m_{0}}-\epsilon-\frac{\epsilon c_{0}}{2}\right) \omega(|t-s|) .
\end{aligned}
$$

Последнее неравенство согласно (28) противоречит тому, что $t, s \in M_{z}$. Таким образом, при условии, что модуль непрерьвности удовлетворяет условию (1), теорема доказана.

Предположим теперь, что для модуля непрерывности $\omega_{0}(h)$ условие $(1)$ не выполнено. Как уже указьвалось вьше, это означает, что $\omega_{0}(h)$ эквивалентно $h$. В этом случае все доказательство сохраняется при замене функции $\bar{\psi}(t)$ на конечнозвенную ломаную без участков постоянства.

Теорема полностью доказана.

Ясно, что если функция $x \in H(\omega)$ исправима, то $\mu\left(M_{x}\right)>0$. Поэтому верно следующее утверждение. 
СлЕДСТВИЕ 1. Множество исправимых функиий в $H(\omega)$ является множеством первой категории в $H(\omega)$.

Пусть $1 \geqslant \beta>\alpha>0$. Поскольку каждая функция из $H_{\alpha}$, для которой найдется функция $y$ из $H_{\beta}$ с $\mu\{t: x(t)=y(t)\}>0$, исправима в $H_{\alpha}$, то справедлив и следующий факт.

СлЕДСТВИЕ 2. Пусть $1 \geqslant \beta>\alpha>0$. Множсество функиий из $H_{\alpha}$, для каждой из которых найдется своя функиия у из $H_{\beta}$, удовлетворяющая условию $\mu\{t: x(t)=$ $y(t)\}>0$, является мнохсеством первой категории в $H_{\alpha}$.

Конечно, следствие 2 содержит и результат К. Гофмана [6], и результат И. М. Михеева [7].

\section{СПИСОК ЦИТИРОВАННОЙ ЛИТЕРАТУРЫ}

[1] Fonf V. P., Gurariy V. I., Kadets M. I. An infinite-dimensional subspace $C[0,1]$ consisting of nowhere differentiable functions // Comp. Rend. Acad. Bulg. Sci. 1999. V. 52. № 11-12. P. 13-16.

[2] Зигмунд А. Тригонометрические ряды. Т. 1. М.: Мир, 1965.

[3] Петрушев П. П., Троянски С. Л. О теореме Банаха-Мазура об универсальности $C[0,1]$ // Comp. Rend. Acad. Bulg. Sci. 1984. T. 37. №3. C. 283-285.

[4] Гурарий В. И. Линейное пространство состоящее из всюду недифференцируемых функций // Comp. Rend. Acad. Bulg. Sci. 1991. T. 44. № 5. C. 13-15.

[5] Rodriguez-Piazza I. Every separable Banach space is isometric to a space of continuous nowhere differentiable functions // Proc. Amer. Math. Soc. 1995. V. 123. № 12. P. 3650-3654.

[6] Goffman C. Approximation of non-parametric surfaces of finite area // J. Math. Mech. 1963. V. 12. P. $737-745$.

[7] Михеев И. М. Одно свойство функций, представимых лакунарными рядами Фурье // Вестн. Моск. ун-та. Матем. -мех. 1972. № 5. С. 67-71.

[8] Бережной Е. И. Оценки равномерного модуля непрерьвности функций из симметричных пространств // Изв. РАН. Сер. матем. 1996. Т. 60. № 2. С. 3-20.

[9] Ульянов П. Л. О работах Н. Н. Лузина по метрической теории функций // УМН. 1985. T. 40. № 3. C. $15-70$.

[10] Brudnyi Yu., Krugljak N. Interpolation Functors and Interpolation Spaces. V. 1. North Holland, 1991. 